\title{
Preface
}

B. Kaiser*, L. Frotscher* and W. Jaegermann*

\section{DFG priority program SPP 1613 “Fuels Produced Regeneratively Through Light-Driven Water Splitting"}

Clarification of the Elemental Processes Involved and Prospects for Implementation in Technological Concepts

https://doi.org/10.1515/zpch-2020-1067

The priority program SPP 1613 "Fuels Produced Regeneratively Through LightDriven Water Splitting" by the German Science foundation (DFG) was initiated during a meeting of a number of researchers from all over Germany at the Reisensburg near Ulm in April 2010. The proposal was accepted by the DFG in early 2012, and the program started at the kick-off meeting taking place in Pommersfelden in November 2012.

$1^{\text {st }}$ funding period: 01.10.2012 - 30.09.2015

$2^{\text {nd }}$ funding period: 01.10.2015 - 31.03.2019

\section{Introduction}

A further use of fossil energy sources as oil, gas and coal, which ensured energy supply for mankind in the past, is no longer possible. On the one hand resources are limited, on the other hand the inherent $\mathrm{CO}_{2}$ emissions may have fatal consequences for the world's climate. Therefore, the creation of a sustainable energy generation and supply from regenerative sources is overdue.

In future energy systems hydrogen $\left(\mathrm{H}_{2}\right)$ technology will most probably play a decisive role, in case an efficient conversion from $\mathrm{H}_{2} \mathrm{O}$ using regenerative power sources can be realized. From $\mathrm{H}_{2}$ as a starting point also chemical fuels can be

\footnotetext{
*Corresponding authors: B. Kaiser, L. Frotscher and W. Jaegermann, Institute for Materials Science, Technical University Darmstadt, 64287 Darmstadt, Germany, e-mail: kaiser@surface.tu-darmstadt.de (B. Kaiser); frotscher@surface.tu-darmstadt.de (L. Frotscher); jagermann@surface.tu-darmstadt.de (W. Jaegermann)
} 
formed in the gaseous or the liquid state (e.g. methane or methanol), which are easier to handle for mobile use. Using such "solar fuels" transport and/or storage issues usually related to the use of volatile solar or wind energy are principally solved.

To produce hydrogen in large amounts and in an environmentally compatible way, the light-driven splitting of water is a promising strategy. However, the artificial systems identified till today do not reach sufficient efficiencies, or are far too expensive for industrial implementation. This is due to the fact that the highly complicated electrochemical processes involved have not been fully understood, yet. And for this reason, the applied materials and device structures still do not operate at their physico-chemical limits. Efficient artificial photosynthesis can only be realized by considering a number of elementary processes and tuning them in correlation to each other in a rational and feasible way:

- Broadband light absorption

- Optimized charge carrier generation and separation

- Loss-minimized electrocatalytic production of $\mathrm{H}_{2}$ and $\mathrm{O}_{2}$ from $\mathrm{H}_{2} \mathrm{O}$ in separated compartments

The objectives of the priority program therefore ranged from a fundamental exploration of involved elementary processes of catalytic $\mathrm{H}_{2} \mathrm{O}$ splitting by dark and photoreactions to a knowledge-based evaluation and investigation of engineering strategies for technological solutions. The challenge was to couple semiconductors for efficient light absorption and separation with metallic, solid or biomimetic catalysts for the $\mathrm{H}_{2}$ (HER) and $\mathrm{O}_{2}$ (OER) evolution reaction directly from $\mathrm{H}_{2} \mathrm{O}$. Within this concept the different individual projects of the priority program were selected to produce synergies by merging their different and complimentary expertise and approaches in collaborative efforts. The coordinator project provided the coordinative and integrative tools to accomplish this duty.

\section{Scientific questions}

The understanding of the involved elementary steps in photoelectrosynthetic water splitting as well as its realization using semiconductors as most promising absorber materials was the main focus of the SPP 1613.

In the first funding period the priority was on the development and analysis of defined model systems for the understanding of (photo)electrochemical reactions at interfaces. Optimized semiconductor systems allow high quantum efficiencies (close to 100\%) in the light-induced electron-hole pair separation. However, neither the contact generation nor the resulting charge carrier dynamics at 
semiconductor/metal/electrolyte interfaces with catalytic coatings (porous metal layers up to metal particles in the nanometer region) have been well understood, yet. The same applies for the following electrochemical multi electron transfer reactions. The model systems in the different areas should be selected in a way, that they allow the analysis of single elementary steps in their complementarity to each other. From a combination of the insights the basic understanding of the system as total may be advanced to a new level. In the first funding period the SPP's main research topics were:

\section{Electrocatalytic systems}

Novel electrocatalysts, which are preferentially composed of cheap and abundant materials, need to be identified and optimized in its coupling to the semiconductor substrates to effectively catalyse the electrochemical reactions to hydrogen and oxygen and avoid high overpotentials.

\section{Photocatalytic systems}

The requirements on photocatalytic systems include the identification of photocatalysts with a reduced bandgap of less than $3 \mathrm{eV}$ and high quantum efficiencies for electron-hole pair separation.

\section{Photoelectrochemical systems}

The research in this area focuses on novel materials and material combinations, which provide the photopotential for effective water splitting, but also represent stable and cost-effective solutions.

\section{Model systems}

To gain atomic-scale insights into the elementary processes occurring during the photoelectrochemical/catalytic water splitting, one or more suitable model systems need to be identified and studied in very detail both by means of experiment and theory.

In the second funding period the central research topic was to identify the scientific material preconditions for further investigations of technologically promising systems, which may serve as basis for the subsequent necessary technological developments. Systems that can be of importance for a technical implementation shall be identified and characterized. Central issue is here the selection of absorbers, which provide a sufficient photovoltage to drive the coupled hydrogen and oxygen evolution reactions (HER, OER). Furthermore, the different components shall be incorporated to demonstrator devices and be analysed with regard to their interaction. For both fields the important scientific material parameters on an atomic/molecular level shall be identified and correlated with the technologically usable macroscopic characteristics and performance levels. 
Another issue was the development or advancement of experimental and theoretical methods for the focused analysis of (photo)electrochemical phenomena in model and real systems. The novel experimental methods and approaches shall deliver extensive information about the involved elementary processes in the examined systems. This can be achieved by the combination of electrochemical procedures with spectroscopic or microscopic methods. An extension towards time dependent measurements of charge carrier dynamics combined with efficiency analyses is still to be sought. An important research goal is also the development of theoretical models to provide an understanding of the basic principles of electrochemical phenomena in photocatalytic systems on different time and length scales. By developing and coupling of appropriate methods in multi-scale approaches the complex step from model to real system shall be approached. Thereby, compared to the experimental analyses reliable statements shall be possible about the working principles of photosynthetic and catalytic systems. From these results knowledge-based optimization strategies are to be deduced. The theoretical simulations contribute to close the gap between idealized model systems and the complex composite materials usually applied in real devices. In the same way, they are of help to build a bridge between classical surface and interface physics in the UHV and the interface processes in electrochemical environment.

Therefore, in the second funding period the subdivision of the SPP was adapted to three different but related project areas that have been studied in close relation to each other to reach the needed and essential synergy effects in the priority program:

\section{Novel photoabsorbers}

Novel absorber materials based on cheap and widely available semiconductors in our case metal oxides and -nitrides are investigated for the applicability in water splitting systems. These can be used in combination with other semiconductorbased electrodes to provide the necessary combined photovoltage $>1.8 \mathrm{~V}$ for water splitting. These materials are to be optimized with regard to charge carrier mobility and stability.

\section{Advanced electrocatalysts}

Promising materials, which have been identified during the first period of the priority program, are to be further optimized with regard to efficiency and stability. Additionally, the underlying mechanisms of their catalytic activity are investigated in detail in order to enable the synthesis of even more efficient or stable catalysts most preferentially based on non-critical elements. Ultimately, they have to be coupled with photoabsorbers to test them in photoelectrochemical cells under real-world working conditions. 


\section{Device development}

Here, all the different material systems of the other working areas need to be combined and tested and optimized as a whole. Standard material for light to electron-hole pair conversion with adjusted photovoltages were different thin film Si multijunction cells. Efficient SPP-made noble metal free electrocatalysts are to be coupled to successful absorber materials in order to demonstrate the feasibility and to characterize the performance properties of an integrated device at an early stage. Special emphasis will be given to the aspects of long-term stability and on separated hydrogen and oxygen producing compartments.

\section{Conclusions}

During the funding periods of the SPP there have been a number of international collaborative efforts and meetings on the subject of light induced photoelectrosynthetic approaches to solar fuels, which can be taken as profound evidence for the scientific, technological, and societal relevance of the topic. Scientists who were involved in the SPP were and are still taking active part in these activities. However, it must also be notified that the research work and the results performed within the SPP, but also in all the very many international research efforts, have still not led to final, well defined technological perspectives. It is fair to argue that the basic understanding of the needs and future research goals for realizing an efficient synthetic artificial leaf approach to a sustainable solar driven fuel generation has strongly improved within the last years most probably also due to the results and success obtained of the priority program. On the other hand, it seems clear that there are still a number of research topics open to be investigated on the fundamental level in understanding the physical and chemical boundary conditions involved in efficient artificial photosynthesis but even more on a technologically motivated level related to the materials science issues and the engineering of working devices.

As within the SPP 1613 we have gathered very many different PIs with always different approaches and priorities in the definition of their research topics, we will refrain to give a summary of the most important results as these will also be a matter of taste and there is still no general agreement on the most prospective route to artificial solar fuel generation. Therefore, as the interested reader will notice, we present some of the main results of all projects within the SPP in this special issue of the "Zeitschrift für Physikalische Chemie". We are confident that the SPP's funding by the DFG was an important step forward for the establishment of the research on artificial fuels in Germany and we do expect a number of further efforts in this decisive research field in the next future. 
The coordination board members thank all the participants (DFG program managers, PIs, postdocs, PhD students and board members) for their fruitful cooperation and support to make the SPP 1613 a great success.

\section{Organisational issues}

In order to support and give advice to the different projects an international advisory committee consisting of outstanding scientists in the field was selected, which joined all the summer schools and meetings of the SPP.

\subsection{Summer schools and report meetings}

Yearly report meetings were scheduled, to which also the referee's and advisory board's members were invited. Special highlights were certainly the summer schools taking place in Ellwangen from the $6^{\text {th }}$ to the $9^{\text {th }}$ of October 2013 and in Berlin-Schmöckwitz from the $26^{\text {th }}$ to the $28^{\text {th }}$ of October 2016, where internationally renowned researchers in the field were invited to give overview lectures in their field of expertise.

Furthermore, three international conferences were organized in the framework of the SPP. The $1^{\text {st }}$ took place near the end of the $1^{\text {st }}$ funding period: International Conference on Light Driven Water Splitting Using Semiconductor Based Devices (SolarFuel15) Mallorca, Spain, in March 2015, in cooperation with NanoGe with a total of approx. 130 international participants, 19 invited and 28 contributed talks and 51 poster contributions. The $2^{\text {nd }}$ meeting took place as NanoGe fall meeting in Torremolinos, Spain, in October 2018 during the $2^{\text {nd }}$ funding period. It was shared with the meeting organized by S. Boettcher and K. Sivula (in total approx. 100 participants). The third and concluding conference took place at the end of the official funding period of the DFG in April 2019 in Taormina, Sicily (see Figure 1). It was organized with support from the Deutsche Bunsengesellschaft für Physikalische Chemie (DBG) and the Gesellschaft deutscher Chemiker (GDCh) as an International Bunsen discussion meeting. In order to broaden the points of discussion to similar research topics the meeting was jointly organized with the project coordinators of the BMBF project "Mangan" and the EU project "A-LEAF" Professors Robert Schlögel (FHI Berlin and MPI CEC Mülheim) and Jose Ramon Galan (ICIQ Tarragona). The International Bunsen discussion meeting had a total of approx. 140 international participants, 29 invited and 46 contributed talks and 56 poster contributions. The SPP contributions are summarized in the present special issue of "Zeitschrift für Physikalische Chemie". 


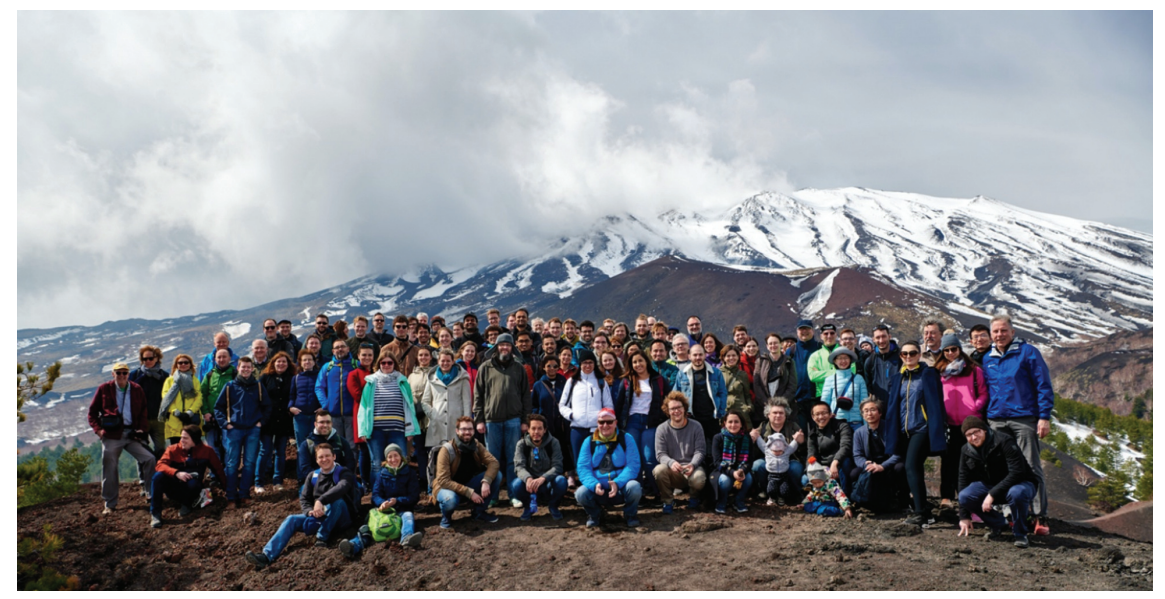

Fig. 1: Participants of the Int. Bunsen Discussion meeting in Taormina on excursion to mount Etna.

Coordination board of the SPP:

Prof. Dr. W. Jaegermann (speaker)

PD Dr. B. Kaiser

L. Frotscher

Homepage: http://www.solarh2.tu-darmstadt.de/solarh2/index.en.jsp

Members of the advisory board

Ib Chorkendorff, Technical University of Denmark

Bruce Parkinson, University of Wyoming, USA

Laurie Peter, University of Bath, UK

Günter Schmid, Siemens AG, Erlangen

John Turner, formerly NREL, Golden, Colorado, USA (retired)

Participating groups in the $2^{\text {nd }}$ funding period

- Ferrites for photoelectrochemical water splitting (Prof. Dr. Detlef Bahnemann, Hannover, Prof. Dr. Thomas Bredow, Bonn, Prof. Dr. Michael Wark, Oldenburg)

- Metal oxide nanostructures for electrochemical and photoelectrochemical water splitting (Prof. Dr. Thomas Bein, München, Prof. Dr. Dina Fattakhova-Rohlfing, München, Prof. Dr. Rossitza Pentcheva, Duisburg, Prof. Dr. Christina Scheu, Düsseldorf)

- Novel thin film composites and co-catalysts for visible light-induced water splitting (Prof. Dr. Malte Behrens, Essen, Prof. Dr. Anna Fischer, Freiburg, Prof. Dr. Martin Lerch, Berlin, PD Dr. Thomas Schedel-Niedrig, Berlin) 
- Development of optimum bandgap photoanodes for tandem watersplitting cells based on doped complex metal oxides and III-V semiconductors coupled to water oxidation electrocatalysts (Prof. Dr. Radim Beránek, Bochum, Prof. Dr. Anjana Devi, Bochum, Dr. Rainer Eichberger, Berlin)

- Development of catalysts, namely manganese oxides and molybdenum sulphides, for an implementation in a light-driven water-splitting device using a multi-junction solar cell (Prof. Dr. Holger Dau, Berlin, Prof. Dr. Sebastian Fiechter, Berlin, Prof. Dr. Philipp Kurz, Freiburg)

- High-throughput characterization of multinary transition metal oxide and oxynitride libraries. New materials for solar water splitting with improved properties (Prof. Dr. Sebastian Fiechter, Berlin, Prof. Dr.-Ing. Alfred Ludwig, Bochum, Prof. Dr. Wolfgang Schuhmann, Bochum)

- Photoelectrochemical water splitting using adapted silicon based semiconductor multi-junction cell structures (Dr. Friedhelm Finger, Jülich, Prof. Dr. Wolfram Jaegermann, Darmstadt, PD Dr. Bernhard Kaiser, Darmstadt, Prof. Dr. Rolf Schäfer, Darmstadt)

- In situ environmental TEM studies of electro- and photo-electrochemical systems for water splitting (Prof. Dr. Christian Jooß, Göttingen)

- Quantum chemical and quantum dynamical studies of the photocatalytic water splitting on titanium dioxide surfaces (Prof. Dr. Thorsten Klüner, Oldenburg)

- Photolectrochemical application of Uranium oxides for enhanced Light Absorption (PECULIAR) (Prof. Dr. Sanjay Mathur, Köln)

- Sustainable solar energy conversion with defined ferrite nanostructures (Dr. Roland Marschall, Bayreuth)

- Zn-doped Gallium Oxynitride Nanoparticles as Efficient Photocatalyst for Water Splitting (Prof. Dr. Martin Muhler, Bochum, Prof. Dr. Markus Winterer, Duisburg)

- Ta3N5 nanotubes and -rods: doping, band-gap engineering and stabilization (co-catalysis) (Prof. Dr. Patrik Schmuki, Erlangen-Nürnberg)

- Nanostructured mixed metal oxides for the electrocatalytic oxidation of water (Prof. Dr. Peter Strasser, Berlin, Dr. Detre Teschner, Berlin)

- Three-dimensional semiconductor nanowire networks as model systems to study physical processes in nanostructured electrodes for light-driven water splitting (Dr. Maria Eugenia Toimil-Molares, Darmstadt)

- Photoelectrocatalytic Anion Substituted Perovskites PAP (Prof. Dr. Anke Weidenkaff, Darmstadt) 